\title{
Digestion in vitro de la polyméthionyl-caséine par les enzymes du tractus digestif
}

\author{
par \\ H. GAERTNER et A. PUIGSERVER \\ Avec la collaboration technique d'Andréa GuIDoNI
}

\section{Ré s u m é}

L'utilisation du N-carboxyanhydride de la méthionine nous a permis de fixer un ou plusieurs résidus d'amino/acide sur chaque groupement aminé de quelques protéines alimentaires modèles : caséines, $\beta$-lactoglobuline, isolats de pois et de fèverole. Dans des conditions favorables à la polymérisation du réactif sur les protéines ( $\mathrm{pH}$ 6,5), le rendement de fixation est voisin de $80 \%$, l'enrichissement en poids de méthionine dépasse $20 \%$ et la taille moyenne des polymères de 6 ou 7 résidus. Le degré de modification des résidus de lysine, très reproductible d'une protéine à l'autre, est voisin de $40 \%$.

Des expériences de digestion " in vitro " par la trypsine et la chymotrypsine montrent que les vitesses initiales d'hydrolyse sont d'autant plus faibles que les chaines de polyméthionine sont importantes et bien réparties sur la protéine. Cependant, la digestion plus poussée des protéines modifiées par la pepsine, le suc pancréatique et l'aminopeptidase intestinale montre que la méthionine fixée est libérée au même degré que les autres aminoacides. L'hydrolyse efficace des liaisons méthionyl-méthionine des polymères associée à celle déjà démontrée par ailleurs de la liaison isopeptidique $\varepsilon$-méthionyl-lysine, laisse prévoir une bonne utilisation " in vivo » des protéines modifiées par fixation covalente d'acides aminés essentiels.

Mots clés

Méthionine - Polyméthionine - Caséines - Digestion - Isopeptide.

Centre de Biochimie et de Biologie moléculaire du C.N.R.S., 31, chemin JosephAiguier - 13277 Marseille cedex 9 (France). 
Titre abrégé

Digestion de polyméthionyl-caséines.

\section{S u m m a ry}

IN VITRO DIGESTION OF POLYMETHIONYL-CASEIN BY ENZYMES OF THE DIGESTIVE TRACT

Methionine, a limiting essential amino acid, has been attached covalently to a number of food proteins including casein and $\beta$-lactoglobulin by the $N$-carboxyanhydride condensation reaction. Proteins are modified via both the a-amino group of the polypeptide chain and the E-amino group of lysyl residues. Under polymerization conditions $(p H 6,5)$, the method can give up to $80 \%$ incorporation of the amount of amino acid derivative used and a final content in methionine of as much as $20 \%$. The average chain length of the polymers is about six or seven amino acids while the extent of modified lysyl residues is about $40 \%$.

The decrease in initial rates of "in vitro "hydrolysis of casein derivatives may be correlated with the chain length and distribution of methionine polymers on proteins. However, extensive hydrolysis by pepsin, pancreatic juice and porcine aminopeptidase $N$ gave rise to an equal relative release of all the amino acids. The efficient hydrolysis of methionyl-methionine bonds by several digestive enzymes, in addition to the already reported hydrolysis of the isopeptide bond E-methionyl-lysine be aminopeptidase, is consistent with the bioavailability of covalently attached methionine polymers.

Key words

Methionine - Polymethionine - Caseins - Digestion - Isopeptide.

\section{INTRODUCTION}

La méthionine, acide aminé essentiel, a pu être greffée sur des protéines alimentaires par la méthode des N-carboxyanhydrides (Bjarnason-Baumann et al., 1977 ; Gaertner, 1981; Puigserver et al., 1982). Ce type de réactif peut en effet se polymériser sur les groupements aminés des protéines en solution (Glazer et al., 1962), mais il a surtout été utilisé pour préparer, en phase organique anhydre, des polymères d'aminoacides de haut poids moléculaire (Stahman, 1962). Le greffage d'acides aminés aux protéines a l'avantage indéniable de permettre la fixation d'une quantité importante de réactif, même si la matrice polypeptidique est pauvre en résidus de lysine. L'aminoacide ainsi fixé est biologiquement utilisable (BjarnasonBaumann et al., 1977; Puigserver et al., 1978). 
Après avoir déterminé les conditions optimales de polymérisation de la méthionine sur la caséine, la méthode a été appliquée à différentes protéines connues pour être déficientes en cet aminoacide. Il devenait donc intéressant d'étudier la digestion des protéines ainsi modifiées par les enzymes du tractus digestif afin de connaître l'influence des polymères greffés sur l'hydrolyse du squelette protéique et avoir une idée de l'utilisation effective de la polyméthionine in vivo.

\section{MATERIELS ET METHODES}

Le N-carboxyanhydride de la méthionine a été préparé par barbotage de phosgène (l'Air Liquide) durant 2-3 h dans une suspension anhydre d'acide aminé (Sigma) dans le tétrahydrofuranne. La température est maintenue à $50^{\circ} \mathrm{C}$ jusqu'à solubilisation complète de l'acide aminé. Après évaporation sous vide du milieu réactionnel, la teneur du produit en $\mathrm{N}$-carboxyanhydride est évaluée par une méthode spécifique (Patchornik et Shalitin, 1961). La caséine (Merck), la B-lactoglobuline (Sigma) et les isolats protéiques de pois et féverole (I.N.R.A.) ont été utilisés sans autre purification. Le suc pancréatique de porc a été fourni par l'I.N.R.A. (Jouy-en-Josas) et l'aminopeptidase intestinale par S. Maroux et al. La pepsine, la trypsine et la chymotrypsine utilisées pour les études in vitro sont des produits Worthington.

La polyaddition de L-méthionine sur la caséine est réalisée en ajoutant une solution de N-carboxyanhydride, dans du tétrahydrofuranne, à une suspension de la protéine à $5 \%$ dans un tampon à $\mathrm{pH}$ 6,5. La protéine modifiée est ensuite dialysée puis lyophilisée et le taux de méthionine déterminé par analyse automatique après oxydation performique. Le degré de modification des résidus de lysine est estimé après guanidination de l'échantillon (Kimmel, 1967). La méthionylcaséine ne contenant qu'un résidu de méthionine par résidu de lysine, a été préparée selon une méthode déjà décrite (Puigserver et al., 1979a).

Les vitesses initiales d'hydrolyse in vitro des protéines par la trypsine et la chymotrypsine sont mesurées en dosant les groupements aminés libres par action de l'acide 2-4-6 trinitrobenzène sulfonique (Fields, 1972), Des concentrations équimolaires de protéines $(4,3 \mu \mathrm{moles} / \mathrm{ml})$ sont incubées avec l'enzyme $(\mathrm{E} / \mathrm{S}=1 / 2000$ en poids) dans du tampon borate de sodium $0,02 \mathrm{M}, \mathrm{pH} 8,2$ à $37^{\circ} \mathrm{C}$. Les digestions par action successive de la pepsine $(\mathrm{E} / \mathrm{S}=1 / 50)$, du suc pancréatique $(\mathrm{E} / \mathrm{S}=1 / 20)$ et de l'aminopeptidase intestinale de porc $(\mathrm{E} / \mathrm{S}=1 / 500)$ ont été réalisées sur des solutions protéiques à $0,5 \%$ dans $\mathrm{HCl} \mathrm{N} / 20$ puis à $\mathrm{pH} 8$ et $\mathrm{pH} 7$ par addition d'un tampon phosphate. Le suc pancréatique de porc est préalablement activé par la trypsine $\left(1 / 20\right.$, en poids) à $\mathrm{pH} 8$ et à $0^{\circ} \mathrm{C}$ durant $3 \mathrm{~h}$. 
Après 10 à $15 \mathrm{~h}$ d'incubation à $38^{\circ} \mathrm{C}$ les différents hydrolysats sont déprotéinisés au TCA à $10 \%$ et analysés directement sur un appareil Beckman modèle $120 \mathrm{C}$.

\section{RESULTATS}

\section{Modification des protéines}

Avec 5 excès de réactif par rapport au nombre de groupements aminés la polymérisation de la méthionine sur la caséine est optimale à $\mathrm{pH}$ 6,5 (fig. 1). La protéine est alors enrichie de $20 \%$ en poids de méthionine, avec un rendement de fixation voisin de $80 \%$. Dans ces conditions, la moitié des résidus lysyl est modifiée et la taille moyenne des polymères de méthionine peut être estimée à

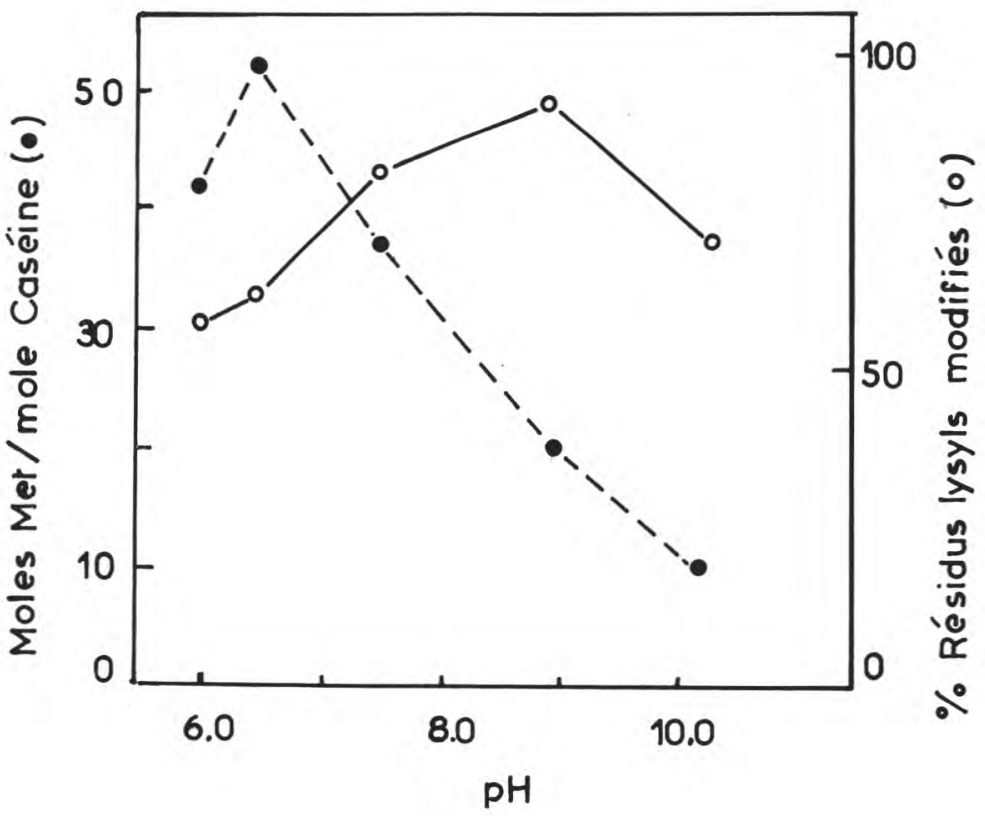

fig. 1

Influence du $\mathrm{pH}$ sur la quantité de méthionine fixée et le degré de modification des résidus lysyl de la caséine. La réaction est faite à $4^{\circ} \mathrm{C}$ avec un rapport molaire NCA-Met/ $\mathrm{NH}_{2}$ égal à 5 dans les tampons citrate de sodium $0,1 \mathrm{M}$ à $\mathrm{pH} 6$ et 6,5 , phosphate de sodium à $\mathrm{pH} 7,5$ et 9 et bicarbonate de sodium à $\mathrm{pH} 10,2$ et 11 .

pH dependence of covalent attachment of methionine to casein and extent of modification of the lysyl residues. The reaction was done at $4^{\circ} \mathrm{C}$ (molar ratio anhydride: amino groups $=5$ ) and the following buffers were used $0.1 \mathrm{M}$ sodium citrate, $p H \quad 6.0$ and 6.5 , sodium phosphate, $p H 7.5$ and 9.0 and sodium bicarbonate, $p H 10.2$ and 11.0. 
6-7 résidus. Appliquée à différents échantillons de protéines alimentaires, cette méthode conduit aux taux d'incorporation portés dans le tableau 1. Le maintien du rapport réactif : $\mathrm{NH}_{2}$ à 4 lors de ces modifications nous permet de comparer les rendements de fixation

\section{TABLEAU 1 - TABLE 1}

Modification de quelques protéines par polyaddition de méthionine à $\mathrm{pH}$ 6,5 (rapport molaire réactif : $\mathrm{NH}_{2}=4$ )

Protein modification by methionine- $N$-carboxy-anhydride under polymerization conditions, $p H 6.5$ with a molar ratio anhydride: amino groups $=4$

\begin{tabular}{|c|c|c|c|c|}
\hline \multirow{2}{*}{ Protéine } & \multicolumn{2}{|c|}{ Composition (\%) } & \multirow{2}{*}{$\begin{array}{c}\text { Lysine } \\
\text { modifiée }(\%)\end{array}$} & \multirow{2}{*}{$\begin{array}{l}\text { Rendement de } \\
\text { fixation }(\%)\end{array}$} \\
\hline & Lys & Met & & \\
\hline$\beta$-lactoglobuline & 12 & 3,2 & - & - \\
\hline Polymet-lactoglobuline & - & 32,0 & 42 & 80 \\
\hline Caséine & 9,0 & 3,0 & - & - \\
\hline Polymet-caséine & - & 22,0 & 43 & 70 \\
\hline Isolat de pois & 6,1 & 0,4 & - & - \\
\hline Polymet-isolat & - & 18,0 & 40 & 73 \\
\hline Isolat de féverole & 6,0 & 0,4 & - & - \\
\hline Polymet-isolat & - & 19,0 & 41 & 75 \\
\hline
\end{tabular}

et la répartition des polymères sur les différentes protéines. Les résultats, très reproductibles d'une protéine à l'autre, montrent que le rendement de fixation est de 70 à $80 \%$ et le degré de modification des résidus de lysine voisin de $40 \%$.

\section{Digestion par la trypsine et la chymotrypsine}

Les vitesses initiales d'hydrolyse par la trypsine et la chymotryp. sine de solutions équimolaires de caséines modifiées à différents degrés sont données dans le tableau 2. Le greffage d'aminoacides ou de polypeptides diminue le nombre de liaisons accessibles. Les vitesses obtenues sont d'autant plus faibles que les chaînes de polyméthionine sont importantes et mieux réparties sur la protéine. 


\section{TABLEAU 2 - TABLE 2}

Vitesses initiales d'hydrolyse des dérivés de la caséine par la trypsine et la chymotrypsine

Relative initial rates of hydrolysis of casein derivatives by trypsin and chymotrypsin

\begin{tabular}{l|c|c|c|c}
\hline \multirow{2}{*}{ Protéine } & $\begin{array}{c}\text { Méthionine } \\
\text { moles/mole }\end{array}$ & $\begin{array}{c}\text { Lysine } \\
\text { modifiée (\%) }\end{array}$ & \multicolumn{2}{|c}{ Vitesses initiales (\%) } \\
\cline { 3 - 5 } & 0 & 0 & 100 & 100 \\
\hline Caséine témoin & 10 & 100 & 52 & 53 \\
Méthionyl-caséine & 11 & 40 & 32 & 34 \\
Polyméthionyl-caséine & 37 & 50 & 18 & 14 \\
Polyméthionyl-caséine & 0 & & & \\
\hline Ti : trypsine ; ChTi : chymotrypsine.
\end{tabular}

\section{Digestion par la pepsine, le suc pancréatique et l'aminopeptidase $\mathbf{N}$}

L'action successive de ces enzymes, qui reproduit la chronologie des événements hydrolytiques in vivo, permet d'étudier la digestibilité des protéines modifiées ainsi que la disponibilité des résidus de méthionine greffés et des résidus de lysine modifiés. Les résultats du tableau 3 laissent supposer une bonne disponibilité de la plupart des acides aminés des protéines modifiées. L'utilisation exclusive de l'aminopeptidase $\mathrm{N}$ n'entraîne qu'une faible libération d'acides aspartique et glutamique, acides aminés les plus représentés dans la protéine native. Par contre, la méthionine est libérée en quantité 5 à 10 fois plus importante que dans la caséine témoin. Ces résultats confirment donc que la méthionine greffée par une liaison isopeptidique dans le cas de la méthionyl-caséine, ou sous forme de polymères dans le cas de la polyméthionyl-caséine, peut être facilement libérée au cours de la digestion enzymatique.

\section{CONCLUSION}

Le greffage d'acides aminés essentiels à une protéine par la méthode des $\mathrm{N}$-carboxyanhydrides a l'avantage de permettre la fixation en une seule étape d'un nombre important de résidus. La polymérisation de la méthionine sur la caséine se traduit par un enrichissement en méthionine qui peut être supérieur à $20 \%$ et une substitution partielle des groupements $\varepsilon$-aminés (40 à $50 \%$ ). La 


\section{TABLEAU $3-T A B L E 3$}

Aminoacides libérés ( $\mu$ moles/ $\mu$ mole protéine) au cours de la digestion par la pepsine, le suc pancréatique et l'aminopeptidase $\mathrm{N}$ de porc de la caséine et de ses dérivés

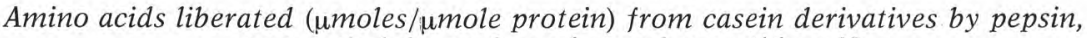
pancreatic juice and porcine aminopeptidase $N$

\begin{tabular}{|c|c|c|c|c|}
\hline & Amino acides & Caséine témoin & $\begin{array}{l}\text { Méthionyl- } \\
\text { caséine (1) }\end{array}$ & $\begin{array}{l}\text { Polyméthionyl- } \\
\text { caséine (2) }\end{array}$ \\
\hline & Asp & 1.5 & 1.1 & 1.1 \\
\hline & Thr + Ser & 11.5 & 10.5 & 11.0 \\
\hline & Glu & 1.3 & 1.3 & 1.0 \\
\hline & Pro & 1.5 & 1.2 & 0.8 \\
\hline & Gly & 1.8 & 1.7 & 1.2 \\
\hline & Ala & 4.1 & 3.8 & 2.9 \\
\hline & Val & 3.6 & 4.3 & 3.6 \\
\hline & Met & 2.4 & 12.4 & 25.4 \\
\hline & Ile & 3.3 & 3.3 & 2.9 \\
\hline & Leu & 10.0 & 10.2 & 9.5 \\
\hline & Tyr & 5.3 & 5.6 & 5.4 \\
\hline & Phe & 5.3 & 5.7 & 5.2 \\
\hline & His & 2.5 & 2.4 & 2.1 \\
\hline & Arg & 3.7 & 3.4 & 3.5 \\
\hline & Lys & 7.7 & 5,9 & 5.9 \\
\hline & \multicolumn{4}{|c|}{$\begin{array}{l}\text { Dérivé de la caséine comportant un résidu méthionyl par résidu de lysine } \\
\text { Dérivé à } 37 \text { moles de méthionine fixées par mole de caséine. }\end{array}$} \\
\hline
\end{tabular}

présence de ramifications peptidiques sur le squelette protéique diminue l'accessibilité des liaisons hydrolysables du squelette initial. La diminution de la solubilité et les phénomènes d'agrégation observés avec la polyméthionyl-caséine y contribuent également pour une large part.

Au cours d'une digestion poussée des protéines par les enzymes du tractus digestif, les acides aminés sont libérés au même degré 
dans les protéines témoin et modifiées. La méthionine fixée est presque totalement libérée. Dans le cas de la méthionyl-caséine, c'est l'aminopeptidase intestinale qui hydrolyse spécifiquement la liaison isopeptidique $\varepsilon$-méthionyl-lysine (Puigserver et al., 1979b). Avec la polyméthionyl-caséine, on montre que les liaisons méthionylméthionine au sein des polymères sont également bien hydrolysées.

Cette étude laisse donc supposer une bonne utilisation digestive de la méthionine polymérisée sur une protéine. Des expériences nutritionnelles devront cependant confirmer nos résultats in vitro. Nous essayons par ailleurs de préciser l'action des enzymes responsables de la digestion des polymères greffés.

\section{Remerciements}

Ce travail a été réalisé grâce à une aide de la Délégation Générale à la Recherche Scientifique et Technique (Action Technologie Alimentaire et Agricole 81.C.0305).

\section{Bibliographie}

Bjarnason-Baumann (B.), Pfaender (P.), Siebert (G.) (1977). - Enhancement of the biological value of whey protein by covalent addition into peptide linkage of limiting essential amino acids. Nutr. Metab., 21, 170-171.

FIELDS (R.) (1972). - The rapid determination of amino groups with TNBS. Meth. Enzym., 25 B, 464-468.

GAertner (H.) (1981). - Polyaddition de la L-Méthionine sur des protéines alimentaires: Thèse de docteur-ingénieur, Université d'Aix-Marseille I.

Glazer (A. M.), Bar-Eli (A.), Katchalski (E.) (1962). - Préparation and characterization of polytyrosyl trypsin. J. Biol. Chem., 237, 1832.

Kimmel (J. R.) (1967). - Guanidination of proteins. Meth. Enzym., 11, 584-589.

PATCHORNIK (A.), SHALITIN (Y.) (1961). - Titrimetric method for continuous determination of carbon dioxide and its application in amino acid chemistry. Analyt. Chem., 33, 13.

Puigserver (A. J.), Sen (L. C.), Feeney (R. E.), Whitaker (J. R.) (1979 a). Covalent attachment of amino acids to caseins. 2, Bioavailability of methionine and $\mathrm{N}$-acetyl methionine covalently linked to casein. $J$. Agric. Food Chem., 27, 1286-1293.

Puigserver (A. J.), Sen (L. C.), Feeney (R. E.), Whitaker (J. R.) (1979 b). Hydrolyse enzymatique et stabilité en milieu acide des liaisons isopeptidiques de la méthionyl-caséine. Ann. Biol. Anim. Bioch. Biophys., 19, 749-755.

Puigserver (A. J.), Gaertner (H.) (1982). - Covalent attachment of essential amino acids to protein by chemical methods: nutritional and functional significance. Adv. Chem. Ser., 1982, 148-167.

Stahman (M. A.) (1962). - Poly- $\alpha$-aminoacids, polypeptides and proteins. Univ. Wisconsin Press, Madison. 